\title{
Documentos
}

\section{Coherencia sistémica e identitaria en mujeres con vaginismo primario}

\author{
Andrés Moltedo-Perfetti ${ }^{1}$, Bernardo Nardi ${ }^{2}$, Emidio Arimatea ${ }^{3}$. \\ ${ }_{1}^{1}$ Psicólogo, Escuela de Psicología, Universidad Santo Tomás, sede Viña del Mar, Chile. \\ ${ }^{2}$ Psiquiatra, ${ }^{3}$ Psicólogo, Centro Adolescenti - Clinica Psichiatrica - Dipartimento di Medicina Clinica e Sperimentale, \\ Università Politecnica delle Marche, Ancona, Italia.
}

\section{RESUMEN}

El vaginismo es una disfunción sexual femenina que genera gran impacto en la relación de pareja. En el presente artículo se busca describir, desde la psicología cognitiva post-racionalista, aquellas características identitarias y de personalidad observadas en 50 mujeres diagnosticadas con vaginismo primario sin antecedentes de alteraciones físicas o ginecológicas y con una historia de vida sin traumas ni abusos sexuales. De acuerdo a lo observado, se trata de mujeres con una positiva visión de sí mismas, que se orientan hacia la consecución del control entre dos necesidades típicamente contrapuestas, la de ser protegidas y la de sentirse libres e independientes. Por ello tienden a centrarse en la regulación de la distancia entre acercamiento y alejamiento respecto de los demás, sobrecontrolando sus emociones. Así, el vaginismo constituiría una forma en la cual se limita la intromisión y la constricción por parte del otro, a la vez que se evita la dependencia del mismo, y se ejerce control sobre el compromiso emocional mediante la no entrega completa de sí misma durante la relación sexual.

PALABRAS CLAVE: Vaginismo, disfunción sexual, identidad, post-racionalismo

\section{SUMMARY}

Vaginismus is female sexual dysfunction that generates big impact on the relationship. The present article seeks to describe, from the post-rationalist cognitive psychology, those identity and personality characteristics observed in 50 women diagnosed with primary vaginismus without history of physical or gynecological disorders and a history of life without trauma or sexual abuse. According to observations, it is women with a positive view of themselves, which are oriented towards control between two typically conflicting requirements, that of being protected and to feel free and independent. Therefore tend to focus on the regulation of the distance between rapprochement and estrangement from others, overcontrolling their emotions. So, vaginismus constitute a form in which the intrusion is limited and constrained by the other, while avoiding dependence thereof, and exercised control over emotional engagement through full delivery not herself during intercourse.

KEY WORDS: Vaginismus, sexual dysfunction, identity, post-rationalism 


\section{INTRODUCCIÓN}

Si bien el estudio y el interés científico por la sexualidad y la patología sexual ha existido desde hace siglos, es recién a partir de mediados del siglo $\mathrm{XX}$ que ha surgido un interés con características realmente científicas, a partir de los trabajos de Kinsey $(1,2)$ y de Masters y Johnson (3-6).

Las disfunciones sexuales pueden ser definidas como trastornos donde los problemas psíquicos y/o físicos dificultan la ejecución o la satisfacción en la actividad sexual $(7,8)$. Los criterios para su diagnóstico por parte del DSM IV-TR (9) son los siguientes:

A) Aparición persistente o recurrente de espasmos involuntarios de la musculatura del tercio externo de la vagina, que interfiere el coito. B) La alteración provoca malestar acusado o dificultad en las relaciones interpersonales. C) El trastorno no se explica mejor por la presencia de otro trastorno mental (por ejemplo, trastorno de somatización) y no es debido exclusivamente a los efectos fisiológicos directos de una enfermedad médica.

Así, el vaginismo es una contracción muscular involuntaria del tercio externo de la vagina (o primer tercio de la misma), específicamente del esfínter vaginal, que impide la penetración y la realización del coito (10). Este persistente espasmo ocurre independientemente del grado de excitación sexual de la mujer, pues se produce en el momento que la mujer prevé que se va a producir el intento de penetración por su gran temor al dolor (11), siendo un espasmo condicionado por la fobia a la penetración que se asocia cognitivamente a dolor y, por supuesto, todo intento de penetración en estas condiciones va a ser doloroso, por lo que refuerza la disfunción sexual (12).

Como se aprecia, para que una disfunción sexual sea diagnosticada como tal, requiere que las dificultades en el funcionamiento sexual ocurran de manera persistente y que sean causa de malestar psicológico en la persona y/o que genere problemas en la pareja (13).

Acorde a la intensidad de la contracción, se puede clasificar en diversos grados: severo cuando la intensidad de la contracción impide la entrada a la vagina; moderado, cuando el nivel de intensidad de la contracción es menor, permitiendo parcialmente la penetración; y leve cuando se puede producir la penetración, pero con dificultad y muchas veces con dolor (14). Por otra parte, se puede clasificar al vaginismo de acuerdo a desde cuándo se viene generando, es decir, es primario o de toda la vida cuando se da desde el primer intento de penetración; y secundario o adquirido cuando aparece tras un período en el que se dio un funcionamiento penetrativo y/o coital sin dificultades (9).

Así, en el vaginismo primario la mujer nunca ha podido realizar el coito, pero con excepción del mismo, en el resto de la vida íntima, estas mujeres pueden tener una sexualidad satisfactoria, siendo capaces de lograr el orgasmo con diferentes actividades y tipo de estimulación. Al momento de asistir a la consulta pueden llevar años de actividades sexuales en pareja, pero acuden debido a los sentimientos de culpa ante la imposibilidad de la penetración, cosa que típicamente resulta insatisfactoria para su pareja, o de quedar embarazadas $(6,15)$.

En el caso del vaginismo secundario las dificultades para realizar la penetración aparecen tras un período en el cual la mujer ha podido realizar el coito aparentemente sin dificultad en diversas ocasiones. También es posible encontrar mujeres en las que el vaginismo aparece de forma intermitente en unas situaciones, pero no en otras, o sólo en unas determinadas posiciones de coito.

La prevalencia del vaginismo oscila entre un $2 \mathrm{y}$ un $4 \%$ de las mujeres adultas $(6,16)$, aunque algunos autores llegan a referir hasta un $7 \%$ de las mismas (17). Su incidencia en el número de consultas oscila entre un $5 \%$ y un $20 \%$ de las mujeres que acuden por padecer dificultades sexuales (18-22), pero puede que se encuentre sub-reportada debido a lo sensible del trastorno, ya que existe un número significativo de mujeres que no manifiestan sus dificultades ni siquiera con su ginecólogo, ni buscan atención clínica, o asiste a un variado número de especialistas $(17,23,24,25)$.

En la bibliografía se reporta una mayor frecuencia en mujeres de nivel educativo y socioeconómico alto (26). Si bien en la literatura se reportan algunos factores físicos asociados a la aparición o a la mantención del vaginismo, como la existencia de un himen rígido e inflexible, la endometriosis, la inflamación pélvica, o la presencia de otros trastornos u otras enfermedades o tumores, los factores preponderantes son de origen psicológico $(5-8,15,22,27)$.

La mayor parte de los autores refiere que desde el punto de vista psicológico, el vaginismo se produce por un proceso fóbico de aprendizaje asociado al miedo a la penetración, y se mantiene por la ansiedad experimentada por la mujer ante la proximidad de la misma (6). En este proceso influyen la existencia de creencias de que la penetración puede causar dolor y heridas, así como miedos al embarazo, al nacimiento, al SIDA y otras enfermedades de transmisión sexual (ETS) $(8,27)$.

Se puede observar un modelo donde en los factores predisponentes y de mantención del vaginismo se aprecian temáticas como los roles sexuales, las conductas evitativas y la ansiedad $(28,29,30)$, la que muchas veces se acepta como la principal causa del vaginismo $(15,27,31,32)$. Se trata de una respuesta condicionada y anticipatoria del dolor, de temor y ansiedad, que se aprecia no sólo respecto de la actividad sexual, sino que puede apreciarse, también, durante la exploración ginecológica, o en la imposibilidad del uso de tampones durante la 
menstruación, asumiendo tener una vagina más corta y estrecha que las demás mujeres y, especialmente, respecto del largo y diámetro del pene, el espéculo o los tampones $(15,16,19,30,31)$. Esta disonancia cognitiva les lleva a asumir como imposible o muy dolorosa la penetración, incluso con el temor o fantasía de ser rasgadas o partidas por el pene (27). Experiencias sexuales traumáticas, como una violación, así como el dolor experimentado en la primera relación sexual o la anticipación del mismo en aquella, también son señalados como causantes de vaginismo $(27,28)$.

Diversas investigaciones clínicas $(25,28,29,33,34)$ señalan que las mujeres con vaginismo suelen tener una percepción negativa de la sexualidad, especialmente respecto de las relaciones sexuales prematrimoniales. También se ha señalado como frecuente una educación sexual y religiosa estricta y culpógena, asociada a la idea del coito como pecado, con fuertes creencias respecto de qué es lo correcto o incorrecto en la vida, en general, y en lo sexual, en particular $(6,8,14,16)$. Así, al estudiar la sexualidad humana y, específicamente, disfunciones sexuales como el vaginismo, resulta imprescindible centrarse no sólo en el estudio de la respuesta sexual en sí. Es esencial incorporar las características subjetivas y la historia de la mujer que vive, experimenta y expresa dicha sintomatología o sexualidad, ya que aquella no consiste sólo en la aptitud de un individuo para lograr una respuesta erótica, sino que es una dimensión completa de la personalidad (6).

Siguiendo esa línea, se buscó ver qué características identitarias comunes mostraba un grupo de mujeres adultas chilenas que asisten a terapia psicológica, diagnosticadas con vaginismo primario, sin antecedentes de alteraciones físicas o ginecológicas y con una historia de vida sin traumas ni abusos sexuales.

\section{PROCESOS DE IDENTIDAD}

La complejidad cerebral y/o mental no puede ser reducida sólo al número de neuronas y sus relaciones, a la complejidad física del sistema nervioso central o a la de su estructura anátomo-química, sino que también, y principalmente, a cómo la mente construye un conocimiento de sí y del mundo (35). Es más, la complejidad de cada sistema cognitivo individual radica en la capacidad para auto-referirse de manera estable, lo que le permite generar una identidad de sí mismo constante, a pesar de las permanentes presiones de la experiencia inmediata vivida y de los subsecuentes cambios y transformaciones del ciclo vital $(35,36)$. El ser humano puede ser entendido como un sistema autoorganizado, autopoieticamente cerrado, que se desarrolla a lo largo de su ciclo vital manteniendo de manera constante la unidad y la continuidad de su identidad como sistema $(35,36,37)$.
Siguiendo ese desarrollo teórico $(38,39,40)$, el Sí mismo se aprecia en una permanente dialéctica con circularidad recursiva, entre la ipseidad y la mismidad (41). Mientras la ipseidad puede ser vista como la construcción de la identidad propia sobre la sintonización con los referentes externos, la mismidad será la construcción de la propia identidad sobre la interioridad y sobre el mantenimiento del sentido de sí (42), siendo la parte del sí mismo que se mantiene invariante y que aporta el sentido de unicidad personal y continuidad a pesar del transcurso temporal.

Por lo tanto, cada individuo a lo largo de su ciclo vital mantiene su manera peculiar de estructurar sus estados internos $(43,44)$, efectuando un orden subjetivo de su experiencia de acuerdo con un marco referencial en el que se decodifican, asimilan y reordenan los eventos vividos en la experiencia inmediata. Así, la organización individual resulta invariante y determina las características esenciales del mismo, permitiendo la estabilidad, mientras que la estructura sistémica puede ser modificada en la interacción con el ambiente (45), generando, a lo largo del ciclo de vida individual, formas absolutamente únicas e irrepetibles de acoplamiento estructural.

El sí mismo surge en un proceso organizativo continuo que acontece y se va desarrollando con sentido de unicidad sistémica coherente a lo largo del ciclo vital, permitiéndole al individuo mantener su propia coherencia interna, percibiéndose a sí mismo como una persona única y diferente de todas las demás al asimilar las perturbaciones y oscilaciones que emergen del nivel tácito ("Yo"), así como sus nuevas estructuraciones, que debieran ser explicitadas en la imagen consciente en curso ("Mí") (46).

Durante el transcurso vital de todas las personas, surgen en la experiencia inmediata una serie de eventos discrepantes que pueden ser percibidos como perturbaciones $(35,36)$. Por esta razón, toda historia personal debe ser desarrollada, articulada e incluso modificada constantemente para que mantenga su consistencia frente a las perturbaciones del fluir experiencial (47).

\section{ASPECTOS CARACTERÍSTICOS}

Las mujeres con vaginismo se caracterizan por un sentido de sí mismas ligado a modalidades cognitivas que pueden ser descritas como concretas y con bajos niveles de abstracción (47-51), privilegiando la operatividad práctica respecto a la especulación, orientándose hacia la consecución del control entre dos necesidades típicamente contrapuestas, la de ser protegidas por una figura que representa una base segura y la de sentirse libres $\mathrm{e}$ independientes en un mundo que es percibido como potencialmente peligroso. 
Las separaciones respecto de las personas percibidas como base segura o referencias confiables, así como del espacio de libertad y autonomía, tienden a ser percibidas inicialmente con miedo y ansiedad y, con posterioridad, suelen ser "somatizadas".

De esa manera, se aprecia una importante tendencia para responder en términos de miedo y ansiedad, como también una disminución de las conductas autónomas (40). El miedo, no sólo es una de las emociones básicas (52-55), sino que constituye la emoción prototípica del script narrativo $(56,57)$ de las mujeres con esta sintomatología. Así, el miedo será un refuerzo constante en la búsqueda de conductas de apego, contacto y de búsqueda de proximidad con la figura cuidadora, así como de bloqueo de la conducta exploratoria (58).

El sentido de sí mismas se encuentra dado por la tendencia concreta (49) y marcadamente sensorial ligada a contextos y situaciones específicas (59). Estas mujeres tienden a centrarse en la regulación de la distancia entre acercamiento y alejamiento respecto de las figuras significativas de referencia $(60,61)$, así como de los contextos ambientales que son vividos como familiares (62). En ambos parámetros tienden a ejercer un control constante $(63,64)$.

Emerge, de esa manera, la necesidad de sentirse adecuadamente protegidas sin perder el contacto y la protección por parte de la figura protectora y reasegurante $(65,66)$, así como la necesidad de desplegarse en el ambiente y de sentirse libre de constricciones, limitaciones y vínculos de distinta naturaleza (67). Aquellas situaciones en las cuales esto no es posible, son vividas como circunstancias que escapan de su control y que pueden resultar potencialmente peligrosas o perturbadoras $(68,69)$.

\section{LA ACTITUD HACIA SÍ MISMAS, LOS OTROS Y LA REALIDAD}

La actitud hacia sí mismas es en términos generales positiva, vencedora y exitosa, pero solamente en los casos y momentos en los que tienen bajo control a las personas y ambientes que les dan seguridad y confianza (49). A la par, existe un deseo de ponerse a prueba mediante la búsqueda de libertad debido a un esquema tácito de tipo constrictivo (69).

Otro componente presente en la imagen de sí mismas es la sensación intrínseca de debilidad, ya sea física o mental, la cual limita las potencialidades positivas, transformándose en la explicación de cada evento y sensación negativa. Presentan una oscilación recursiva entre polaridades antitéticas, una de protección y otra de necesidad de libertad, lo cual le permite al sentido de sí mismas el percibirse como agente controlador, ya sea brindándose autoconfianza o sentimientos de autonomía e inde- pendencia en la confirmación de la habilidad de ser capaces de controlar las relaciones interpersonales (62).

Así mismo, es observable una tendencia a reaccionar intensa y emotivamente frente a situaciones de amenaza de separación (pérdida de protección) o de incrementos de compromiso emocional (pérdida de libertad), percibidas o reales (66). Construyen, dentro de estos procesos oscilantes, una sensación de identidad estable mediante una atribución externa, donde las emociones no son vistas como resultado de su propio estilo afectivo personal, sino como confirmaciones de la existencia de una realidad coercitiva y peligrosa.

La actitud de estas mujeres consigo mismas se aprecia en la manera en que intentan sobrecontrolar tanto sentimientos como emociones, los cuales son vistos como externos y se los pretende evitar al máximo $(38,39,49,69)$. De la misma manera, al enfrentarse con emociones que pueden generar un desbalance afectivo, el sobrecontrol puede llevar a la exclusión selectiva de experiencias desafiantes, así como una desconexión cognitiva entre la respuesta mental respecto de la situación interpersonal que la causó (70).

En el caso de mujeres que realicen exclusiones completas de las experiencias, su propia conducta aparece es descrita como inentendible los eventos que las gatillan, por lo que ante la imposibilidad de autorreferírselas, son atribuidas a causas externas a sí mismas, típicamente quejas somáticas o psicológicas $(39,64)$. Por una parte, estas mujeres buscan obtener proximidad aseguradora por parte de ciertas figuras consideradas como protectoras, en tanto que evitan la atemorizadora experiencia que les significa la limitación de su libertad de acción (69). Así, el sobrecontrol respecto de los otros se efectúa mediante la atención selectiva de características formales así como metacomunicacionales de las interacciones, más que al contenido específico de las interacciones. Mantienen, por una parte, un rol estable de las redefiniciones de las relaciones, manipulándolas acorde a las necesidades de protección o de control.

Esta actitud de control se intensifica en las relaciones íntimas, ya que éstas habitualmente suscitan emociones más intensas y comprometedoras. Así, el estilo afectivo de estas mujeres se caracteriza, por una parte, por el constante intento de tener una figura de referencia a su disposición, mientras que por otra, por el intento de evitar sentimientos de dependencia y limitaciones a la propia libertad.

La actitud sobrecontroladora respecto de sí mismas y del entorno, emerge progresivamente con posterioridad a la resolución adolescente, constituyendo la estrategia básica para la mantención del equilibrio dinámico de las necesidades opuestas de libertad y protección (71). Aquello se plasma en la búsqueda de relaciones afectivas que provean de 
un sentido de protección, junto con una incapacidad para dominar los aspectos emotivos del afecto humano, concentrándose en aspectos más bien formales de las relaciones humanas, así como el control inter e intrapersonal.

En la adolescencia, el surgimiento del pensamiento abstracto les ha llevado a sentirse protagonistas activas de la realidad, buscando prevenir y limitar aquellas posibles debilidades o posibles incapacidades frente a aquellos eventos y situaciones que son vividas como peligrosas. La estrategia prototípica de enfrentamiento ante estos peligros "objetivos" es la utilización del control de todas las experiencias en las que puede sentirse dependiente y/o débil (71).

El vaginismo puede constituir una forma en la cual se limita la intromisión y la constricción por parte de otro, se evita la dependencia. La constricción en una relación significativa, puede fácilmente producir sentimientos y experiencias emocionales cuyo control puede requerir la elaboración de explicaciones más complejas que aquellas permitidas por la actitud controladora del individuo. Así, resulta relevante mantener el control sobre el compromiso emocional mediante la no entrega completa de sí misma durante la relación sexual. Uno de los grandes problemas o peligros consiste en dejarse llevar por las sensaciones corporales, específicamente aquellas relativas al acto y al placer sexual (71).

En estas descompensaciones psicosexuales juega un rol clave el temor y los riesgos vinculados con la exploración sexual, con el alejamiento de los vínculos protectores familiares, con la posibilidad de contraer enfermedades de transmisión sexual o de vincularse en situaciones constrictivas, de las cuales es difícil si no imposible salir (71). Se activan, por tanto, procesos tácitos, de los cuales el vaginismo es la expresión conductual, permitiendo recuperar la dificultad en situaciones percibidas como constrictivas 0 , por el contrario, cuando la pareja es percibido como imprescindible base segura, se reclama la atención y el cuidado mediante los síntomas, probando de esta manera la confiabilidad, la disponibilidad y los sentimientos e intenciones del compañero.

Así, se observan discrepancias entre un sentimiento de soledad emergente y/o de constricción, con la imagen consciente aceptada de sí misma como agente controlador (47). Conscientemente experimenta sentimientos que le resultan intolerables, como el miedo a perder el control y a las consecuencias asociadas a dicha pérdida de control, ya sea a que se genere una adicción sexual, o a las sensaciones y/o activaciones corporales, como el aumento del ritmo coronario, etc.

Para mantener su auto-imagen, típicamente elaboran teorías respecto de enfermedades que explican sus dificultades (35). Dichas explicaciones de debilidad no reducen o afectan la autoestima de estas mujeres, considerándose personas con enfermedades o problemas, pero no "poco queribles". En otras palabras, al otorgarle una connotación de enfermedad aquello que les pasa, logran darse una explicación consistente de sus sentimientos de angustia ante la anticipación del acto sexual, por lo que no resulta necesario modificar su nivel de autoestima (47).

El contenido de las cogniciones antes descritas se pueden observar presentes no sólo en lo relacionado con el ámbito sexual, sino que también lo están en otros momentos de la vida de estas mujeres, las cuales tienden a evitar hacerse conscientes de su vida emocional, a la que refieren tratar de mantener siempre bajo control (36). Las activaciones emotivas, provenientes del nivel tácito, tenderán a manifestarse a través del miedo a perder el control (48). La intimidad sexual, percibida como constrictiva y como pérdida de la libertad individual, se traducirá en la activación automática de sentimientos de temor.

Si la mujer no es capaz de lograr una integración del desequilibrio a la par de un aumento de la auto-conciencia, la patología se mantendrá y podría transformarse en una situación crónica (48). Es decir, esta conducta se estabiliza porque la mujer vuelve a ejercer su actitud sobrecontroladora en la relación, logrando más poder y autonomía mediante el control de la vida sexual, una suerte de balance paradójico y estable relacionado con dominación y sumisión.

\section{PALABRAS FINALES}

La sintomatología surge como consecuencia de experiencias vividas como abandónicas o constrictivas $(38,39)$ y se expresan con un sentido de pérdida del control y con síntomas que a menudo son de tipo físico o "somático" (69), manifestándose en un sector específico del organismo.

El miedo tácito de perder el control se expresa, a nivel explícito, en el temor de estar a merced de la sintomatología, enfermarse, ponerse mal, volverse "loca" o morir. Así, la sensación subjetiva de pérdida de control es referida a los aspectos concretos de su experiencia y es descrita como algo que se teme enfrentar (49).

La sensación de pérdida de control sobre el dominio de la existencia puede ser activado por preocupaciones por la propia salud, por la pérdida de eficiencia física, por el temor de estar mal o de envejecer, por la enfermedad o la pérdida de un coetáneo ("me puede ocurrir también a mí). En otros casos, los factores precipitantes son situaciones afectivas o laborales que dan una sensación de precariedad, de incertidumbre, de poca confianza, de no tener una guía adecuada y protección en un mundo que se le aparece más amenazante que reasegurante, o bien, de hostilidad, de imposición, 
de condicionamiento, de obligación, de constricción para hacer algo que no se quiere hacer (60).

Se trata en todo caso de situaciones que ocurren no por libre elección del sujeto, el cual al contrario las vive como impuestas por lo externo o de cualquier manera como inevitables: si se siente frágil, entonces vacila la seguridad anteriormente poseída, se pierde eficiencia, se ve a merced de los demás, del sistema o de la enfermedad (64). En estos casos el sujeto tiende a activar conductas de evitación de las situaciones temidas y a buscar posibles fuentes de reaseguración por parte de las figuras percibidas como "confiables".

En estos desbalances, la modalidad concreta de pensamiento canaliza la ansiedad a través de síntomas somáticos "objetivamente" sustentados, que expresan el malestar percibido a nivel tácito de manera indirecta y simbólica $(48,50)$. En este caso, el vaginismo se comporta de manera semejante a lo que podría observarse o decirse de las fobias sexuales. Es decir, se trata de modalidades concretas de pensamiento, en momentos en los cuales estas mujeres no pueden evitar afrontar las experiencias perturbadoras (36).

Los eventos perturbadores activan los canales somáticos del miedo y de la angustia, en este último caso, se trata de ansiedad asociada a un componente somático, ante situaciones en las cuales la experiencia sexual en curso es percibida por la mujer como incontrolable y amenazante (35).

\section{CONCLUSIÓN}

El tema explícito del vaginismo es indicativo de la activación tácita de pérdida de control, ligada al sentido de constricción o al de abandono y vulnerabilidad, si bien la mujer no es consciente de ello y refiere sus síntomas a una "enfermedad" de naturaleza orgánica (71).

\section{REFERENCIAS}

1. Kinsey A, Pomeroy W, Martin C. La Conducta Sexual del Hombre. Siglo Veinte, $1^{\text {a }}$ edición, 1967.

2. Kinsey A, Pomero, W, Martin C, Gebhardt P. La Conducta Sexual de la Mujer. Siglo Veinte, $1^{\underline{a}}$ edición, 1967.

3. Masters W, Johnson V. Respuesta Sexual Humana. Inter-Médica. $1^{\mathrm{a}}$ reimpresión, 1978.

4. Masters W, Johnson V. Incompatibilidad Sexual Humana. Inter-Médica, 2 $2^{\text {a }}$ edición, 1981.

5. Masters W, Johnson V. El Vínculo del Placer. Grijalbo, 14ª edición, 1994.

6. Masters W, Johnson V, Kolodny R. La Sexualidad Humana. Grijalbo. 7a edición, 1987.

7. Labrador F. Tratamiento psicológico de las disfunciones sexuales. En: Echeburúa, E. (Ed.) Parejas en conflicto: Estrategias psicológicas de intervención. San Sebastián: Universidad del País Vasco, 1987.

8. Labrador F. Disfunciones Sexuales. Madrid: Funda- ción Universidad Empresa, 1994.

9. American Psyquiatric Association. Manual Diagnóstico y Estadístico de los Trastornos Mentales (DSM-IVTR). Madrid: Masson; 2002.

10. Basson R, Berman J, Burnett A, Derogatis L, Ferguson D, Fourcroy $\mathrm{J}$, et al. Report of the international consensus development conference on female sexual dysfunction: Definitions and classifications. J Urol 2000;163:888-93.

11. Basson R. Human sex-response cycles. J Sex Marital Ther 2001;27:33-43.

12. Basson R, Leiblum S, Brotto L. Revised definitions of women's sexual dysfunction. J Sex Med 2004;1:4048.

13. Bach A, Wincze J, Barlow D. Sexual Dysfunction. En: Barlow, D. (Ed.) Clinical handbook of psychological disorders. New York: The Guilford Press, 2001.

14. Astolfi E. Disfunciones sexuales femeninas: ¿Qué es el vaginismo?; Aprender Salud; HIBA; 2007.

15. Kuile M, Lankveld J, Groot E, Melles R, Neffs J, Zandbergen M. Cognitive-behavioural therapy for women with lifelong vaginismus: Process and prognostic factors. Behav Res Ther 2006;45:359-73.

16. Hurtado F., Teodoro E, Royo A, Muñoz Y. Estudio sobre disfunciones sexuales femeninas: Incidencia por sexos y análisis de variables biopsicosociales predictoras. Cuadernos de Medicina Psicosomática y Psiquiatría de Enlace 1996;37:24-33.

17. Pacik P. Vaginismus: Review of current concepts and treatment using botox injections, bupivacaine injections, and progresive dilation with the patient under anesthesia. Aesthetic Plast Surg 2001;35:1160-4.

18. Mears E. Sexual problems clinics. An assessment of the work of 26 doctors trained by the Institute of psychosexual medicine. London. Public Health London 1978;92:218-23.

19. Bancroft J. Human sexuality and its problems. Churchill Livingstone. Nueva York, 1983.

20. Heisler J. Sexual therapy in the national marriage guidance council. Marriage Guidance Council, Rugby, 1983.

21. Spector I, Carey M. Incidence and prevalence of the sexual dysfunctions: a critical review of the empirical literature. Arch Sex Behav 1990;19:389-96.

22. Lahaie M, Boyer S, Amsel R, Khalife S, Binik Y. Vaginismus: a review of the literature on classification/ diagnosis etiology, and treatment. Woman's Health 2010;6:705-19.

23. Oniz A, Keskinoglu P, Bezircioglu I. The prevalence and causes of sexual problems among premenopausal Turkish women. J Sex Med 2007;4:1575-81.

24. Nusbaum M, Gamble G, Skinner B, Heiman J. The high prevalence of sexual concerns among women seeking routine gynecological care. J Fam Pract 2000;49:229-32.

25. Reissing E, Binik $Y$, Khalife $S$. Does vaginismus exist? A critical review of the literature. J Nerv Ment Dis 1999;187:261-74

26. Kaplan H, Sadock B, Grebb J. Sinopsis de psiquiatría. Ciencias de la conducta. Psiquiatría clínica. Editorial médica panamericana. Madrid, 1996.

27. West S, Vinikoor L, Zolnoum A. A systematic review of the literature on female sexual dysfunction prerevalence and predictors. Annu Rev Sex Res 2004;15:40-172.

28. Reissing E, Binik Y, Khalife S, Cohen D., Amsel R. 
Etiological correlates of vaginismus: Sexual and physical abuse, sexual knowledge, sexual selfschema, and relationship adjustment. J Sex Marital Ther 2003;29:47-59.

29. Reissing E, Binik Y, Khalife S, Cohen D, Amsel R. Vaginal spasm, pain, and behavior: An empirical investigation of the diagnosis of vaginismus. Arch Sex Behav 2004;33:5-17.

30. van der Velde J, Everaerd W. The relationship between involuntary pelvic floor muscle activity, muscle awareness and experienced threat in women with and without vaginismus. Behav Res Ther 2001;39:395408.

31. Bodenmann G, Ledermann T, Blattner D, Galluzzo C. Associations among everyday stress, critical life events, and sexual problems. J Nerv Ment Dis 2006;194:494-501.

32. Kaya B, Unal S, Ozenli Y, Gursoy N, Tekiner S. Anxiety, depression and sexual dysfunction in women with chronic pelvic pain. Sex Relat Ther 2006;21:187-96.

33. Goldstein A, Pukall C; Goldstein I. (eds) (2009) Female sexual pain disorders. Blackwell, New York.

34. Abdo C, Oliveira W, Moreir, E, Fittipaldi J. Prevalence of sexual dysfunction and correlated conditions in a sample of brazilian women: results of the Brazilian study on sexual behavior (BSSB). Int J Impot Res 2004;16:160-6.

35. Moltedo, A. Nardi, B. Processi di mantenimento dell'identità: tra continuità e cambiamento. Quaderni di Psicoterapia Cognitiva 2010;15:98-113.

36. Moltedo A. Procesos de la mantención de la identidad: entre la continuidad y el cambio. Psicoperspectivas 2006;5:35-47.

37. Oneto L, Moltedo A. Las organizaciones de significado personal de Vittorio Guidano. Una llave explicativa de la experiencia humana. Psicoperspectivas 2002;1:8391.

38. Guidano, V. (1991). The Self in Progress. Guilford, New York.

39. Guidano, V. (1987). Complexity of the Self. Guilford, New York

40. Guidano V, Liotti G. (1983). Cognitive Processes and Emotional Disorders. Guilford Press.

41. Ricoeur P. (1990). Soi-même comme un autre. Èditions du Seuil, Paris.

42. Arciero G, Guidano V. (2000). Experience, explanation and the quest for coherence. En Neimeyer, R., Raskin, J. (Eds) Constructions of Disorder. American Psychiatric Association.

43. Damasio A. Sentir lo que Sucede, Andrés Bello, Santiago, 2000.

44. Damasio A. El error de Descartes. Barcelona, Crítica, 2001.

45. Capra F. La trama de la vida. Anagrama, 1998.

46. Mead G. Espíritu, persona y sociedad. Barcelona, Paidós, 1999

47. Moltedo A. Más allá de la nosografía: la explicación cognitivo procesal sistémica del síntoma. Psicoperspectivas 2004;3:85-93.

48. Moltedo A. Le Difficoltà della Nosografia del Disturbi dell'Umore. En B. Nardi (Ed). Disturbi dell'Umore. Ancona: Ed. Accademia Dei Cognitivi Della Marca. Italia, 2007.

49. Nardi B. Processi psichici e psicopatologia nell'approccio cognitivo. Nuove prospettive in psicologia e in psichiatria clinica. Milán: FrancoAngeli, 2001.

50. Quiñones A. Significado Social y Viabilidad Emocional Narrativa. En: Franklin F, Nabuco C (Eds). Constructivismo em Perspectiva: Implicaces Teóricas e Prácticas para la Psicoterapia. Artes Medicas do Sul, 1997.

51. Quiñones A, Zagmutt A. El Síntoma como Distinción Autorreferencial. Revista Terapia Psicológica $N^{\circ} 25$, 1996.

52. Ekman P. Emotions Revealed. Times Books, New York; Weidenfeld \& Nicolson, London, 2003.

53. Ekman P. An argument for basic emotions. Cognition and Emotion, 1992, 6, 169-200.

54. Ekman P. Facial expression of emotions: New findings, new questions. Psychological Science 1992;3:34-8.

55. Ekman P. Facial Expression and Emotion. American Psychologist 1993;48:384-92.

56. Tomkins, S. Script theory: Differential magnification of affects. En: Howe, H., Page M. (Eds), Nebraska Symposium on Motivation. University of Nebraska Press, Lincoln, 1978.

57. Nathanson D. What's a Script. Bulletin of The Tomkins Institute 1996;Volume 3, Spring-Summer.

58. Nardi B. La organización del sí mismo: de la derivación psicopatológica de Guidano al significado adaptativo de construcción de la reciprocidad. Revista de Psicoterapia 2009;19:103-31.

59. Nardi B, Moltedo A. Desarrollo del modelo de las organizaciones de significado personal de Vittorio Guidano: los procesos de construcción del sí en el modelo cognitivo post-racionalista evolutivo. Psicoperspectivas 2009;8:93-111.

60. Nardi B, Capecci I. I processi di organizzazione degli stili di personalità e le basi dell'unicità personale. Quaderni di Psicoterapia Cognitiva 2006;18:48-83.

61. Nardi B, Capecci I. La relazione di reciprocità nello sviluppo delle diverse organizzazioni di significato personale. Quaderni di Psicoterapia Cognitiva 2007; 19:62-89

62. Nardi B, Capecci I. Contributo per una lettura evolutiva e adattiva della organizzazione di significato negli stili di personalità. Quaderni di Psicoterapia Cognitiva 2005; 17:34-52.

63. Nardi B. Rol de los procesos filogenéticos y ontogenéticos en el desarrollo de las organizaciones de significado personal. Psicoperspectivas 2006;5:49-64.

64. Nardi B. La coscienza di sé. Franco Angeli, Milano, 2013.

65. Nardi B, Moltedo A. Rol de la relación de reciprocidad en el desarrollo de las diversas Organizaciones de Significado Personal. Gaceta de Psiquiatría Universitaria, Facultad de Medicina Universidad de Chile, 2008, año 4, Vol 4, No 3, 345-358.

66. Nardi B. Costruir Si. Sviluppo e Adattamento del Sé nella Normalità e nella Patologia. Franco Angeli, Milano, 2007.

67. Nardi B, Bellantuon, C, Moltedo A. Nuevas perspectivas en la psicoterapia Post-Racionalista: una aproximación adaptativo evolutiva a las Organizaciones de Significado Personal (O.S.P.). Gaceta de Psiquiatría Universitaria, Facultad de Medicina Universidad de Chile, 2010, año 6, volumen 6, No 1, pp 63-70.

68. Nardi B, Moltedo A. Processi di costruzione del sé: 
sviluppi del modelo di Vittorio Guidano sulle organizzazioni di significato personale. Quaderni di Psicoterapia Cognitiva 2009; 24:56-71.

69. Reda M. Sistemi cognitivi complessi e psicoterapia. Roma, La Nuova Italia Scientifica, 1986.

70. Bowlby J. El vínculo afectivo, Paidós, 1993.
71. Moltedo A. Le sfide psicosessuali nell'adolescenza. En: B. Nardi (Ed) Normalitá e Disagio nell'Adolescenza. Ancona: Ed. Accademia Dei Cognitivi Della Marca. Italia, 2003. 